\title{
AN IMPROVED TUNABLE FILTER TOPOLOGY FOR HF PRESELECTION
}

\author{
R. Pastore and J. A. Kosinski, U. S. Army CECOM, Ft. Monmouth, NJ \\ H. L. Cui, Stevens Institute of Technology, Hoboken, NJ
}

\begin{abstract}
In the field of military electronics, there remains a need for tunable HF preselectors featuring small size, low power consumption, and reliable operation through environmental extremes. The maturation of SAW and RF component technologies over the past decade have led to a renewed interest in the feasibility of tunable SAW filters for this role. Last year, we discussed these possibilities in the context of the prior literature. In particular, we derived a figure of merit, and discussed the basic operating principles, design goals, and implementation considerations for such a filter. The filter topologies reported in the literature were found to suffer limitations in center frequency tuning range, bandwidth tuning range, and aliasing of the passband during tuning.

We have developed a new filter topology that addresses these limitations. The topology is based upon both input and output IDT frequency tuning in combination with differing IDT synchronous frequencies. In theory, the additional degrees of freedom of this topology allow for an efficient answer to the previous limitations. Broadband center frequency tuning is obtained since the center frequency is no longer limited to a range defined by a fixed input IDT. Improved bandwidth control is obtained trough selective overlap of the input and output IDT transfer functions. Aliasing effects are minimized by proper selection of the nominal input and output IDT center frequencies. In practice, of course, there are still limitations to the performance that can be achieved. In this paper we discuss both the theoretical advantages and practical limitations of the new filter topology.
\end{abstract}

\section{Introduction}

Communications receivers are often faced with the problem of trying to receive a weak communications signal in the presence of one or more strong interfering signals. Whereas the receiver may have sufficient sensitivity to receive the weak signal against a "quiet" background, against a "noisy" background including one or more strong interfering signals the receiver will be desensitized to a level determined by the strength of the largest interfering signal and the dynamic range of the receiver. Consider a "wide-open" receiver whose input is hard limited to a level $P_{\text {sat }}$, and having RF gain $G$, noise figure $F$, and noise power bandwidth $B_{0}$. The detector portion has a tangential signal sensitivity TSS. In a "quiet" RF environment, one can use $G=[T S S-(N+F)]$ for maximum system sensitivity to weak signals. With a "strong" interference signal of $P>\left(P_{\text {sat }}-G\right)$, the AGC will reduce the system sensitivity by $1 \mathrm{~dB}$ for each $\mathrm{dB}$ of $\mathrm{P}>$ $\left(P_{s a t}-G\right)$ up to the hard limit, i.e., there is a potential loss in sensitivity up to value of $\mathrm{G}$.

One can add a front-end filter to the receiver. The filter is characterized by bandwidth B, insertion loss I, and sidelobe (rejection) level $S$. The interference signal power is reduced by $\mathrm{S} \mathrm{dB}$, and thus for $\mathrm{S}>\mathrm{G}$, out-of-band interference does not desensitize the receiver. The power in the signal of interest is reduced by $\mathrm{I} d \mathrm{~dB}$. Thus, compared to the "wide-open" receiver, the sensitivity in a quiet $R F$ environment is degraded by $I+(F / I)-10 \log \left(B / B_{0}\right)$ [which may be a net improvement], but the sensitivity in the presence of strong interference is improved by as much as $\mathrm{G}-\left[\mathrm{I}+(\mathrm{F} / \mathrm{I})-10 \log \left(\mathrm{B} / \mathrm{B}_{0}\right)\right]$ provided that $\mathrm{S}>\mathrm{G}$. The net result is a figure of merit for system performance improvement essentially given by sidelobe level $\mathrm{S}$, which establishes how well the desensitization is obviated, minus the insertion loss 1 , which establishes how much the quiescent performance is degraded. These considerations are driving the development of an improved tunable filter for HF preselection [1].

\section{Topological Considerations}

The ability of the filter to meet the performance goals depends in part on the choice of filter topology, and in part upon the technology used to implement the filter. Filter and device topologies considered previously include IDT arrays, filter banks, variable wave velocity devices, dispersive delay devices, convolvers, correlators, and matched filters, and transversal filters. of these topologies, the SAW-based programmable transversal filter appears best suited for the HF preselection task. 
Partially programmable SAW transversal filters have been examined by several researchers [2-14]. The partially programmable transversal filter topology is illustrated in Figure 1 for the case of real tap weights. A conventional, fixed input IDT is used in conjunction with a tunable output IDT comprised of a finger array (no busbars), tap weight network, and signal summer. Complex tap weights are implemented using two parallel channels coupled through $90^{\circ}$ hybrids at both input and output.

This topology is inherently limited with respect to center frequency and bandwidth selectability. Limitations on center frequency tunability arise from the use of a fixed input IDT, in that low insertion loss can only be obtained for center frequencies falling within the input IDT coupling bandwidth. Limitations on bandwidth tunability arise from the minimum and maximum overall time delays that can be accommodated in a realistic substrate and IDT geometry. Further limitations on bandwidth arise from aliasing effects encountered when using the simpler single channel circuit and real as opposed to complex tap weights.

\section{Improved Topology}

The limitations on center frequency and bandwidth tunability, as well as certain aspects of passband aliasing, can be overcome readily by using a fully programmable filter architecture with different input and output IDT synchronous frequencies. The improved topology is illustrated in Figure 2. It should be noted that employing a tunable input IDT does not of itself resolve the bandwidth tunability or aliasing limitations; the use of different IDT synchronous frequencies is critical to obtaining the advantages of the improved topology.

\section{Comparison of Performance}

The two additional degrees of freedom incorporated in the improved topology allow for an efficient answer to the previous limitations.

\section{Center Frequency Tuning}

As illustrated in Figure 3, broadband center frequency tuning is obtained since the center frequency is no longer limited to the range defined by the coupling bandwidth of the fixed input IDT.

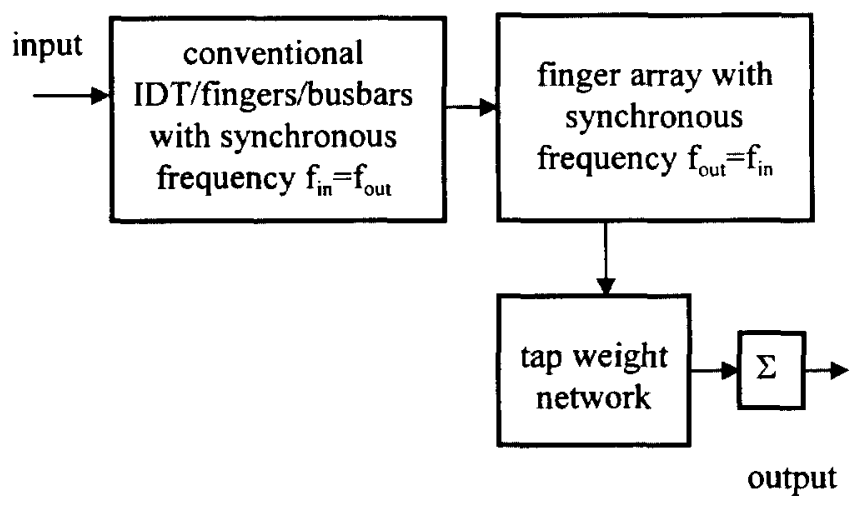

Figure 1. Partially programmable transversal filter topology.

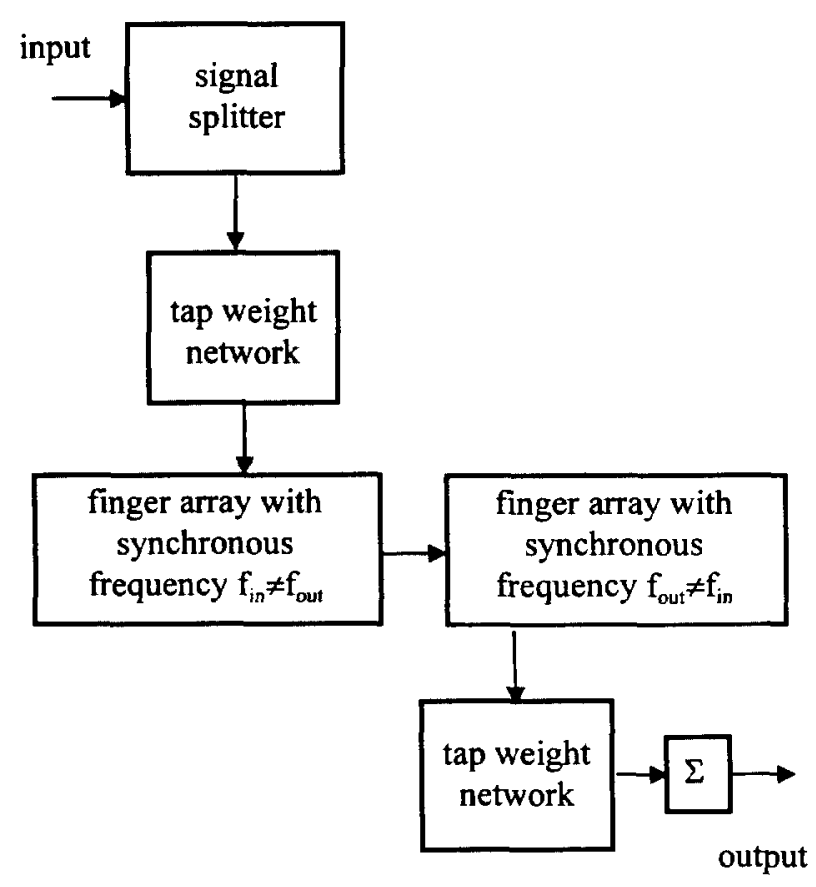

Figure 2. Fully programmable transversal filter topology. 


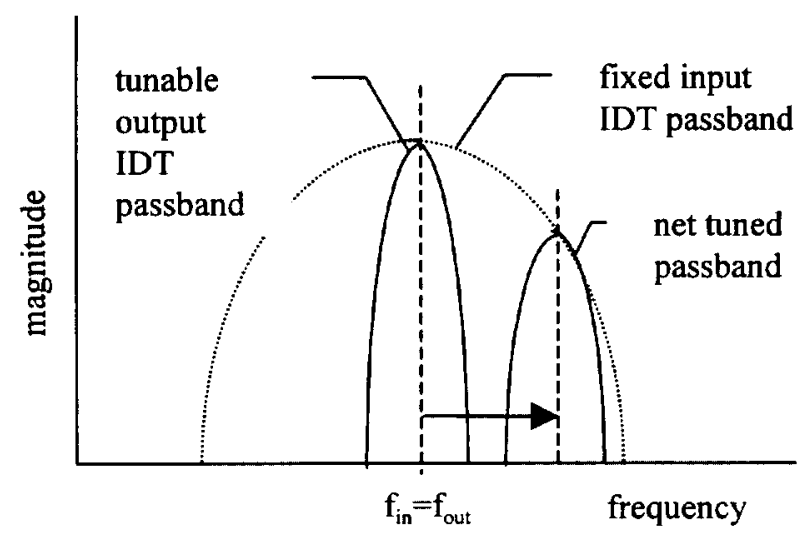

(a)

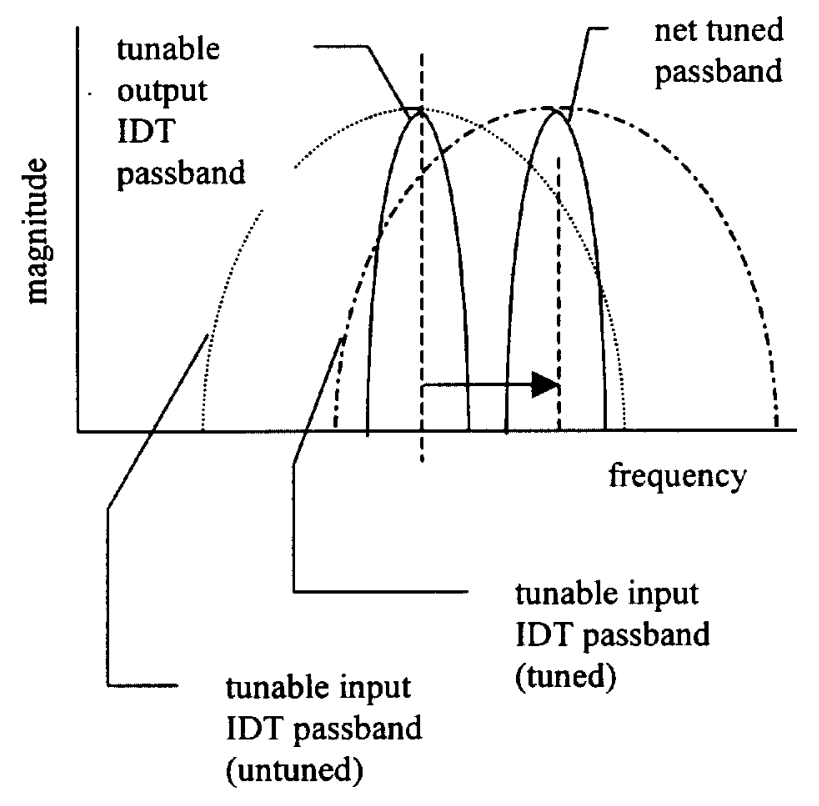

(b)

Figure 3. Center frequency tuning for (a) partially programmable and (b) fully programmable topologies.

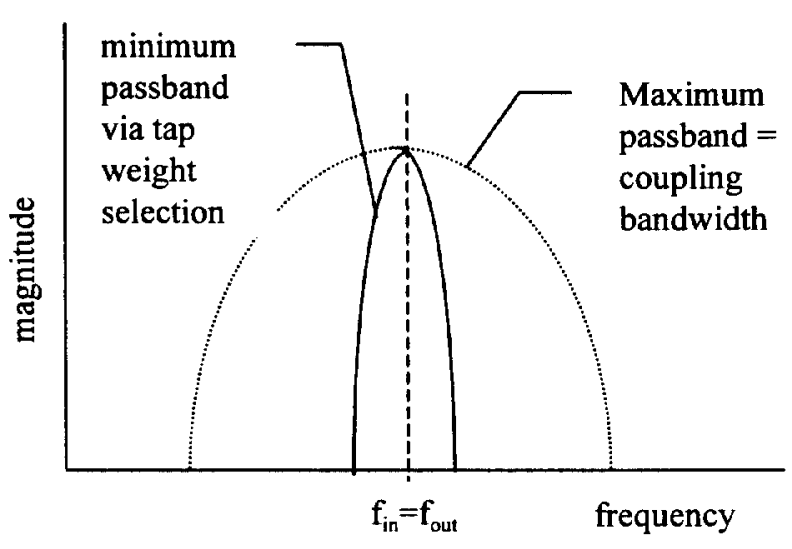

(a)

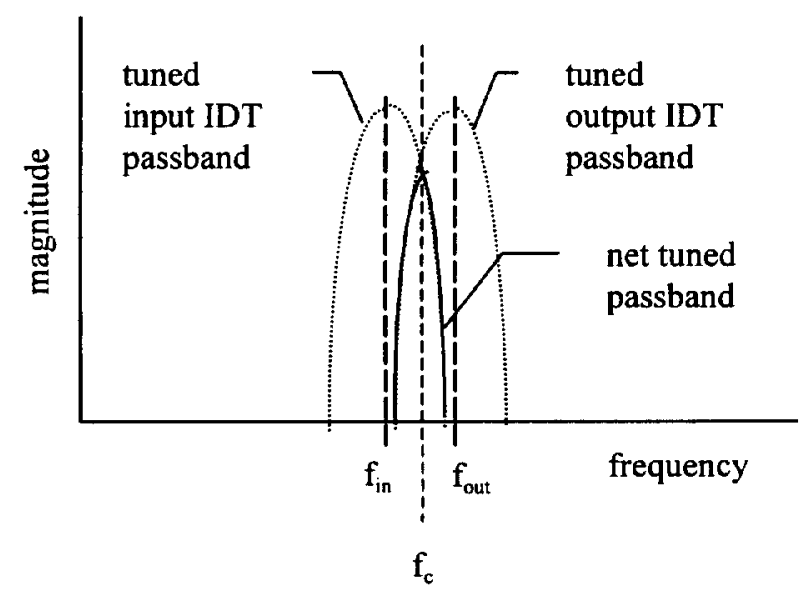

(b)

Figure 4. Bandwidth control for (a) partially programmable and (b) fully programmable topologies.

\section{Bandwidth Control}

The maximum possible filter bandwidth for a given insertion loss is determined by the piezoelectric coupling of the SAW substrate, and the improved topology is unchanged in this regard. However, the improved topology does yield additional flexibility with regard to the minimum possible filter bandwidth. The minimum bandwidth in a SAW transversal filter generally is considered to be the transition bandwidth, which is inversely proportional to the time delay through the SAW device. Thus the physical length of the device fixes the minimum possible bandwidth in a conventional SAW filter. In a partially programmable SAW filter, the bandwidth can be varied either by varying the active device length by turning taps on or off, or through control of the weighting function. In the improved topology, somewhat narrower bandwidths are achievable through selective overlap of the input and output IDT transfer functions. This is illustrated in Figure 4. 
There are two important points to note regarding this option. First, this option requires nonuniform rolloff in the transition band; i.e. the transition band is composed of a shoulder followed by skirts that are somewhat narrower than the total transition bandwidth. This can be achieved through selection of an appropriate weighting function to shape the passband. The narrowest possible bandwidth is then limited by the steepness of the skirts.

The second point is that narrowing the bandwidth incurs a penalty in increased insertion loss. At first glance, this may appear to be a significant drawback, and clearly this is undesirable in applications where the available gain is limited. However, narrowing the bandwidth also reduces the system noise floor at nearly the same rate as the insertion loss is increased, leading to an essentially unchanged signal-to-noise ratio as the bandwidth is narrowed. Hence, where gain is not limited, the selective overlap option provides a viable approach to achieving narrower filter bandwidths than those imposed by the transition bandwidth. By way of illustration, selective overlap using max-rolloff weighting allows a minimum filter bandwidth 10x narrower than the transition band, with $10 \mathrm{~dB}$ of additional insertion loss but unchanged signal-to-noise ratio.

\section{Passband Aliasing}

Frequency translation of the IDT passband is accomplished by incorporating multiplication into the tap weights. Unambiguous frequency translation requires a complex multiplication, which in tum requires a dual channel architecture featuring separate I and $Q$ channels along with broadband $90^{\circ}$ splitters and combiners. The use of a simpler single channel architecture, providing only a real multiplication, leads to passband aliasing, meaning that identical passbands are generated both above and below the nominal IDT center frequency.

The improved topology reduces the aliasing effects by proper selection of the input and output IDT synchronous frequencies as illustrated in Figure 5. Since the input and output IDT synchronous frequencies differ, one set of the aliased passbands overlaps, while the other set of passbands falls out-of-band and is thus strongly attenuated. Provided that the synchronous frequencies differ by more than the coupling bandwidth, only the single IDT out-of-band rejection level limits the reduction in single channel aliasing effects.

\section{Discussion}

The improved topology addresses most of the limitations in the prior art regarding center frequency tunability, bandwidth selectability, and single channel aliasing effects. In addition, the use of substantially differing synchronous frequencies for the input and output IDTs eliminates the component of triple-transit-echo (TTE) arising from mechanical reflections. Unfortunately, the new topology does not address electrical regeneration effects. As a consequence, novel circuitry is being evaluated which would allow selectable impedance matching as dictated by the particular operational scenario. This would allow the selection of a mode featuring minimum insertion loss with maximum TTE (suitable for a

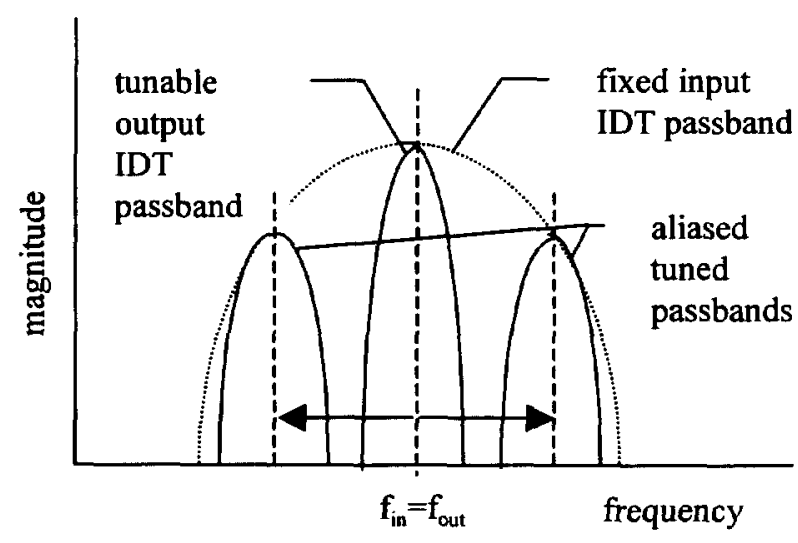

(a)

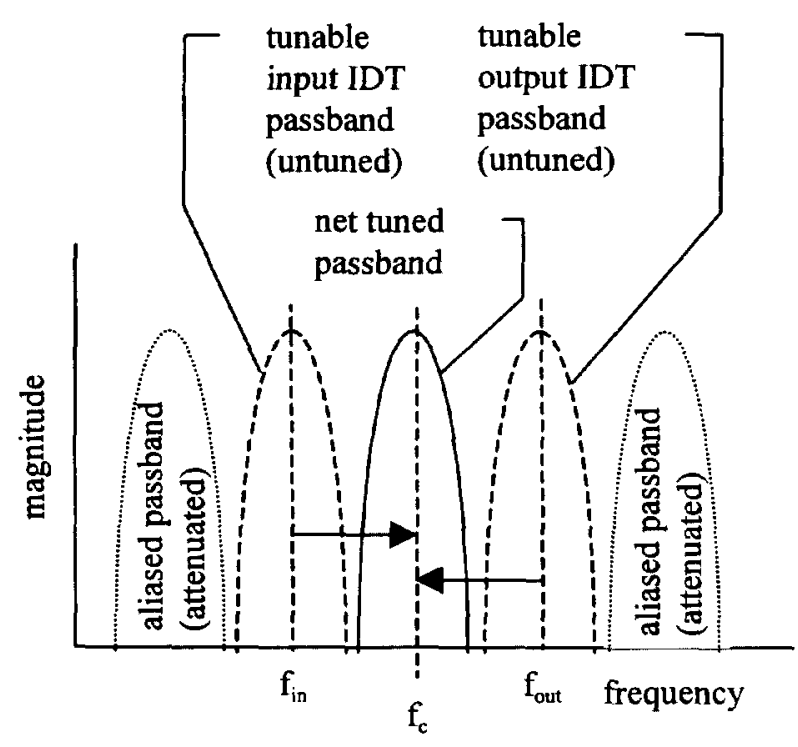

(b)

Figure 5. Aliasing effects for (a) partially programmable and (b) fully programmable topologies. 
pulse detection trigger circuit) or a mode featuring increased insertion loss with reduced TTE (suitable for a communications receiver).

\section{Conclusions}

We have developed a new filter topology for tunable HF preselectors that addresses the limitations in center frequency tuning range, bandwidth tuning range, and aliasing of the passband during tuning reported in the literature. The topology is based upon both input and output IDT frequency tuning in combination with differing IDT synchronous frequencies. Broadband center frequency tuning is obtained since the center frequency is no longer limited to a range defined by a fixed input IDT. Improved bandwidth control is obtained trough selective overlap of the input and output IDT transfer functions. Aliasing effects are minimized by proper selection of the nominal input and out IDT center frequencies. Approaches for minimizing TTE effects are currently being developed.

\section{Acknowledgments}

The authors wish to thank Professor Eric L. Adler of McGill University for significant constructive criticisms of our original technical approach to the selectable performance interference filter. These criticisms forced us to re-evaluate our technical approach, leading ultimately to the improved filter topology presented in this paper. The authors also wish to thank Professor Donald C. Malocha of the University of Central Florida for additional helpful criticisms and Professor William D. Hunt of Georgia Institute of Technology for numerous helpful discussions.

\section{References}

1. R. Pastore, et al., "Design of a Selectable Performance Front End Filter Using Acoustic Surface Wave Resonators," Proc. 1997 IEEE Int'l Freq. Cont. Symp., May 1997, pp. 858-866.

2. P. J. Hagon, et al., "Surface Acoustic Wave Adaptive Transversal Filters," 1974 IEEE USP, Nov. 1974, pp. 177-180.
3. K. M. Lakin, et al., "Adaptive Transversal Filter Synthesis Employing Spatially Orthonormal Eigenvectors", 1974 IEEE USP, Nov. 1974, pp. 172-176.

4. C. M. Panasik, " $250 \mathrm{MHz}$ Programmable Transversal Filter," 1981 IEEE USP, Oct. 1981, pp. 48-52.

5. C. M. Panasik, "SAW Programmable Transversal Filter for Adaptive Interference Suppression," 1982 IEEE USP, Oct. 1982, pp. 100-103.

6. C. M. Panasik and J. R. Toplicar, "Adaptive Interference Suppression Using SAW Hybrid Programmable Transversal Filters," 1983 IEEE USP, Oct.-Nov. 1983, pp. 170-174.

7. D. E. Oates, et al., "Wide-Band SAW/FET Programmable Transversal Filter," 1984 IEEE USP, Nov. 1984, pp. 312-317.

8. J. Y. Duquesnoy, et al., "A Monolithic 7 TapProgrammable Transversal Filter on Gallium Arsenide," 1984 IEEE USP, Nov. 1984, pp. 303307.

9. C. M. Panasik and D. E. Zimmermann, "A 16 Tap Hybrid Programmable Transversal Filter Using Monolithic GaAs Dual-Gate FET Array," 1985 IEEE USP, Oct. 1985, pp. 130-133.

10. D. E. Oates, et al., "SAW/FET Programmable Transversal Filter with 100-MHz Bandwidth and Enhanced Programmability," 1985 IEEE USP, Oct. 1985, pp. 124-129.

11. F. Guediri, et al., "Performance of Acoustic Charge Transport Programmable Tapped Delay Line," 1987 IEEE USP, Oct. 1987, pp. 11-14.

12. D. E. Oates, et al., "SAW/FET Programmable Filter with Varistor Taps for Improved Performance," 1988 IEEE USP, Oct. 1988, pp. 155-158.

13. C. M. Panasik, et al., "A 32 Tap Digitally Programmable Transversal Filter," 1988 IEEE USP, Oct. 1988, pp. 151-154.

14. C. M. Panasik and M. Jurgovan, "Programmable Transversal Filter Based Adaptive Line Enhancer," 1992 IEEE USP, Oct. 1992, pp. 227230. 